\title{
Comparison on epidemiology, tumor location, histology, and prognosis of intracranial germ cell tumors between Mayo Clinic and Japanese consortium cohorts
}

\author{
*Hirokazu Takami, MD, PhD, ${ }^{1,3,4}$ Avital Perry, MD, ${ }^{1}$ Christopher S. Graffeo, MD, MS,1 \\ Caterina Giannini, MD, PhD, ${ }^{2}$ Yoshitaka Narita, MD, PhD, ${ }^{5}$ Yoichi Nakazato, MD, PhD, ${ }^{6}$ \\ Nobuhito Saito, MD, PhD, ${ }^{4}$ Ryo Nishikawa, MD, PhD, ${ }^{7}$ asao Matsutani, MD, PhD, ${ }^{7}$ \\ Koichi Ichimura, MD, PhD, ${ }^{3}$ and David J. Daniels, MD, PhD ${ }^{1}$
}

\begin{abstract}
Departments of ${ }^{1}$ Neurologic Surgery and ${ }^{2}$ Laboratory Medicine and Pathology, Mayo Clinic, Rochester, Minnesota; ${ }^{3}$ Division of Brain Tumor Translational Research, National Cancer Center, Tokyo, Japan; ${ }^{4}$ Department of Neurosurgery, Faculty of Medicine, University of Tokyo, Japan; ${ }^{5}$ Department of Neurosurgery and Neuro-oncology, National Cancer Center Hospital, Tokyo, Japan; ${ }^{6}$ Department of Pathology, Hidaka Hospital, Gunma, Japan; and ${ }^{7}$ Department of Neuro-Oncology/Neurosurgery, Saitama Medical University International Medical Center, Saitama, Japan
\end{abstract}

OBJECTIVE Central nervous system (CNS) germ cell tumors (GCTs) are rare malignant neoplasms that arise predominantly in adolescents and young adults. CNS GCTs demonstrate characteristic trends in national associations, with implications for both tumor incidence and genetics. Although the incidence of CNS GCTs is markedly higher in East Asia than Western countries, direct comparative analyses between these CNS GCT populations are limited.

METHODS In Japan, to facilitate the genomic analyses of CNS GCTs, the Intracranial Germ Cell Tumor Genome Analysis Consortium was established in 2011, and more than 200 cases of GCTs are available for both tumor tissue and clinical data, which is organized by the National Cancer Center (NCC) Japan. At the Mayo Clinic, there have been 98 cases of intracranial GCTs treated by the Department of Neurologic Surgery since 1988. In this paper, the authors compared the epidemiology, clinical presentation including location and histology, and prognosis between cases treated in the US and Japan.

RESULTS There was no significant difference in age and sex distributions between the databases. However, there was a significant difference in the tumor locations; specifically, the frequency of basal ganglia was higher in the NCC database compared with the Mayo Clinic ( $8.4 \%$ vs $0 \%, p=0.008$ ), and bifocal location (neurohypophysis and pineal gland) was higher at the Mayo Clinic than at the NCC $(18.8 \%$ vs $5.8 \%, p=0.002)$. There was no difference in histological subdivisions between the databases. There was no difference in progression-free survival (PFS) and overall survival (OS) of germinoma cases and OS of nongerminomatous GCT (NGGCT) cases treated with chemotherapy and radiation therapy covering whole ventricles. However, PFS of NGGCTs differed significantly, and was better in the NCC cohorts $(p=0.04)$.

CONCLUSIONS There appears to be a differential distribution of GCTs by neuroanatomical location between major geographic and national groups. Further study is warranted to better characterize any underlying genomic, epigenetic, or environmental factors that may be driving the phenotypic differences.

https://thejns.org/doi/abs/10.3171/2019.11.JNS191576

KEYWORDS germ cell tumor; international comparison; tumor location; prognosis; basal ganglia; bifocal; oncology

\footnotetext{
ABBREVIATIONS AFP = alpha-fetoprotein; BTRJ = Brain Tumor Registry Japan; CARE = carboplatin + etoposide; CNS = central nervous system; COG = Children's Oncology Group; GCT = germ cell tumor; HCG = human chorionic gonadotropin; ICE = ifosfamide + carboplatin + etoposide; iGCT Consortium = Intracranial Germ Cell Tumor Genome Analysis Consortium; NCC = National Cancer Center; NGGCT = nongerminomatous GCT; NOS = not otherwise specified; OS = overall survival; PE = cisplatin + etoposide; PFS = progression-free survival; PGC = primordial germ cell; PRISMA = Preferred Reporting Items for Systematic Reviews and Meta-Analysis; WB = whole brain; $W V=$ whole ventricle.
}

SUBMITTED June 7, 2019. ACCEPTED November 27, 2019.

INCLUDE WHEN CITING Published online January 31, 2020; DOI: 10.3171/2019.11.JNS191576.

${ }^{*}$ K.I. and D.J.D. share senior authorship of this work. 


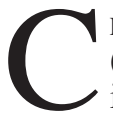
ENTRAL nervous system (CNS) germ cell tumors (GCTs) are rare neoplasms predominantly arising in adolescent and young-adult male patients. Standardized treatment protocols using chemoradiation have demonstrated excellent efficacy, with durable tumor control in $90 \%$ of germinomas. ${ }^{34}$ By contrast, nongerminomatous GCTs (NGGCTs) have proven resistant to a wide array of chemotherapy, radiation, and combined protocols, resulting in a generally poor prognosis. ${ }^{34}$

GCTs demonstrate characteristic racial-geographic distributions, with gonadal GCTs predominating in Western countries, ${ }^{51}$ whereas CNS GCTs arise more frequently in East Asian populations. ${ }^{24,33,43}$ Within the US, the frequency of intracranial GCTs was highest in Asian/Pacific islanders, followed by White and Black populations. ${ }^{46}$ More specifically, while CNS GCTs account for only $5.2 \%$ of primary brain tumors in Western children younger than 14 years of age, ${ }^{43}$ in a comparable Japanese cohort CNS GCTs account for $14.4 \%$, a nearly 3 -fold increase in incidence. ${ }^{38}$ Although genetic or epigenetic factors driving this dramatic differential remain poorly understood, an important discovery was made by Wang et al. in 2015, who utilized whole-genome germline sequencing to identify a key association between a genetic variant of JMJDIc and Japanese CNS GCT, in whom it was 4.8-fold more prevalent, as compared to a matched Western cohort. ${ }^{62}$ JMJDIC is a Jumonji domain-containing protein, which encodes the histone $\mathrm{H} 3 \mathrm{~K} 9$ demethylating enzyme important for removing the trimethyl group on this histone lysine residue, which has been shown to maintain primordial germ cells (PGCs) - the GCT cells of origin - in a murine model. ${ }^{30,42}$ However, it is notable that the variant of this gene has not been proven to be involved in the pathogenesis of GCTs.

To better facilitate large-scale genomic investigations and the identification of targets for novel therapies in these rare and challenging tumors, the Intracranial Germ Cell Tumor Genome Analysis Consortium (iGCT Consortium) was established in 2011. ${ }^{12,13,19}$ At present, the iGCT Consortium has enrolled more than 200 cases including tumor specimens and clinical data, via the National Cancer Center (NCC) in Japan. In parallel, the Mayo Clinic in the US has prospectively registered, biobanked, and followed 98 cases of CNS GCT, including metastatic cases from other organs, that underwent neurosurgical management during the study period (1988-2017).

The goal of the present study was to perform a direct comparison between these large, prospective CNS GCT registries-with particular attention to characteristic differences in demographics, clinical presentation, and treatment response - in hopes of providing new avenues for focused translational inquiry. We report the first pan-Pacific comparison of registry-based CNS GCT cohorts, through which we identified a range of significant differences, including key variations in the distribution of intracranial tumor locations and responses to treatment, particularly among NGGCTs.

\section{Methods \\ Data Source and Acquisition}

Between 1988 and 2017, 80 cases of CNS GCT were neurosurgically managed at the Mayo Clinic. Cases were treated with chemotherapy and radiation therapy by a multidisciplinary team according to the current Children's Oncology Group (COG) guidelines. Eighteen metastatic cases were excluded. Although referral origination data were not universally available, prior data on related samples have shown that approximately $80 \%$ of the patients would be expected to reside in Minnesota or one of the 5 surrounding states comprising the upper Midwest. All histopathological diagnoses were made at the Mayo Clinic, and all pertinent components of the present study were overseen and approved by the IRB.

From 2011 to 2017, 154 primary (e.g., nonmetastatic, nonrecurrent) CNS GCTs treated at 20 participating institutions were enrolled in the iGCT Consortium/NCC database and correspondingly included in this study. There is an overlap of the cases with the previous reports on genomic analyses: 95 of 154 cases were used previously, and the rest were newly employed in this study. ${ }^{12,13,19}$ Cases that were recurrent, metastatic, or diagnosed as non-GCT in the central histopathological review were excluded. According to the Brain Tumor Registry Japan (BTRJ), ${ }^{1}$ the overall annual incidence of GCTs is estimated to be $2.14 \%$. According to the latest results from Cancer Registry and Statistics by the Cancer Information Service, NCC Japan, the number of CNS tumors was 6228 in 2016, which roughly estimates the number of CNS GCTs to be approximately 130 cases per year. Although the proportion of cases captured by the iGCT Consortium cannot be precisely calculated from available data, we estimate that $15 \%-20 \%$ of all Japanese cases are included each year.

In contrast to the above Mayo Clinic cases, patients registered in the NCC database are considered ethnically Japanese people, given their highly homogeneous race background. All histopathological diagnoses were centrally reviewed and reclassified in accordance with the WHO classification of tumors of the CNS by a single expert neuropathologist (Dr. Nakazato). All pertinent aspects of the study were overseen and approved by the NCC Japan Ethics Committee and, as indicated, by each local IRB.

\section{Systematic Literature Review}

A retrospective literature search was conducted using PubMed to capture publications since 1990 reporting outcomes of intracranial GCTs in at least 10 cases per article. This systematic review was performed according to Preferred Reporting Items for Systematic Reviews and Meta-Analysis (PRISMA) guidelines (Fig. 1). PubMed was queried using the key MeSH search terms "neoplasms, germ cell and embryonal," "germinoma," and "central nervous system." Non-MeSH terms were "intracranial" and "germ cell." Citations were screened in all languages, using professional library translation services as needed. All abstracts were reviewed against prespecified criteria by the lead investigator (H.T.), and any candidate citations underwent full-text screening for possible study inclusion. Articles reporting only germinoma or NGGCT were excluded, given the significant association between histology and tumor location. If multiple candidate publications representing the same institutional cohort were identified, the article with the largest cohort was included. Articles not clearly identifying cases as specific to the basal ganglia (e.g., not combined with thalamic cases) were excluded. 


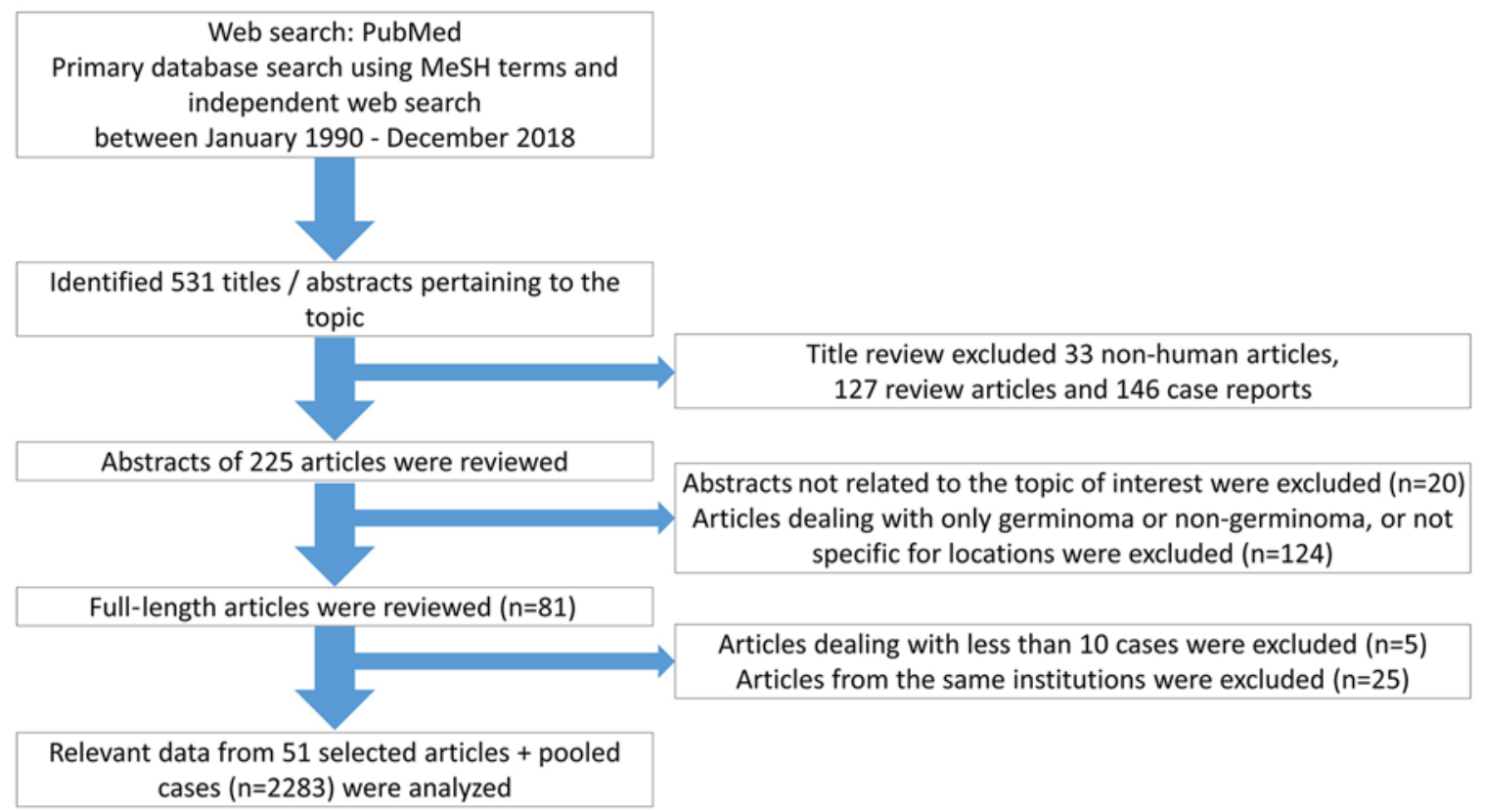

FIG. 1. PRISMA protocol showing the final selection of articles. Figure is available in color online only.

Data abstraction was conducted to capture parallel outcomes to the multicenter series, as available.

\section{Statistical Analysis}

Categorical and continuous variables were analyzed using the Pearson chi-square test and Student t-test, respectively. Nonparametric values were compared using the Wilcoxon signed-rank and rank-sum tests, while time-toevent data were analyzed with the log-rank technique. All statistical analyses were performed using JMP (version 13, SAS Institute Inc.); statistical significance was defined by the type I error rate of $\alpha=0.05$, and all tests were 2-tailed.

\section{Results}

\section{Race, Age, Sex}

Baseline clinical characteristic for all cases are summarized in Table 1. Demographics within the Mayo Clinic cohort included 69 Caucasians, 2 Asians, 1 Latino, 4 Arabs, 1 African American, and 3 unknown. In contrast, the NCC cohort is known to be almost exclusively composed of Japanese patients, a parameter that was not quantitatively verified on a case-by-case basis for this analysis.

Median ages in the Mayo Clinic and NCC databases were 17 and 15 years (ranges $0-56$ and $0-60$ ), respectively, a difference that was not significant $(\mathrm{p}=0.69$; Fig. 2A). Female patients comprised $12(15.0 \%)$ of 80 patients in the Mayo Clinic database and 24 (15.6\%) of 154 in the NCC database, which was not a significant difference $(p=0.91$; Fig. 2B).

\section{Tumor Histology and Markers}

The distribution of histology of the Mayo Clinic database and the NCC database is outlined in Table 1. There were no significant differences in distribution of histologi- cal subtypes across the databases, with the exception of GCTs not otherwise specified (NOS), which were more prevalent in the Mayo Clinic series; however, this most likely reflects a difference in pathologic practice, rather than underlying biology $(\mathrm{p}<0.001$; overall $\mathrm{p}=0.63$; Fig. 2C).

Serum and CSF tested for either human chorionic gonadotropin (HCG) or alpha-fetoprotein (AFP) were compared in germinoma cases between the Mayo Clinic database $(n=36)$ and NCC database $(n=79)$, and no statistically significant difference in tumor markers was observed (Fig. 3).

\section{Tumor Location}

Among Mayo Clinic cases, tumor locations were neurohypophyseal in $17(21.3 \%)$, pineal in $40(50.0 \%)$, bifocal neurohypophysis and pineal in 15 (18.8\%), multiple in 3 (3.8\%), and other in $5(6.3 \%)$. In the NCC database, there were 20 neurohypophyseal (13.0\%), 81 pineal (52.6\%), 9 bifocal (5.8\%), 13 basal ganglia (8.4\%), 22 multiple (14.3\%, including 7 bifocal plus other), and 9 other location (5.8\%) cases. Significant differences in distribution by location included an increased prevalence of bifocal cases in the Mayo Clinic database, and a decreased prevalence of basal ganglia cases $(p=0.002$ and $p=0.008 ;$ Fig. $4 \mathrm{~A})$. When multifocal cases that include bifocal neurohypophyseal and pineal tumors plus another third lesion (7 NCC cases) are counted as bifocal, the difference becomes smaller and nonsignificant $(18.8 \%$ vs $10.4 \%, p=0.07)$. The overall difference of locations between the two databases was statistically significant $(\mathrm{p}=0.0003)$.

\section{Systematic Literature Review of Basal Ganglia GCT Geographic Distribution}

Fifty-one articles met criteria for analysis, resulting in 
TABLE 1. Overview of characteristics of cases in the Mayo and NCC series and their comparison

\begin{tabular}{|c|c|c|c|}
\hline Characteristic & $\begin{array}{l}\text { Mayo Clinic } \\
\quad(n=80)\end{array}$ & $\begin{array}{c}\text { NCC } \\
(n=154)\end{array}$ & p Value \\
\hline Race (White:others) & $69: 8$ & $0: 154$ & \\
\hline $\mathrm{F}: \mathrm{M}$ & $12: 68$ & $24: 130$ & 0.91 \\
\hline $\mathrm{F}, \%$ & 15.0 & 15.6 & \\
\hline \multicolumn{4}{|l|}{ Age, yrs } \\
\hline Range & $0-56$ & $0-60$ & 0.69 \\
\hline Median & 17 & 15 & \\
\hline Mean & 17.6 & 16.9 & \\
\hline \multicolumn{4}{|l|}{ Histology, n \% } \\
\hline Germinoma & $47(58.8)$ & $98(63.6)$ & 0.47 \\
\hline Mature teratoma & $5(6.3)$ & $6(3.9)$ & 0.42 \\
\hline Immature teratoma & $4(5.0)$ & $12(7.8)$ & 0.42 \\
\hline $\begin{array}{l}\text { Teratoma w/ malignant } \\
\text { transformation }\end{array}$ & $0(0)$ & $1(0.6)$ & 0.47 \\
\hline Yolk sac tumor & $1(1.3)$ & $6(3.9)$ & 0.26 \\
\hline Embryonal carcinoma & $0(0)$ & $2(1.3)$ & 0.31 \\
\hline Choriocarcinoma & $1(1.3)$ & $2(1.3)$ & 0.97 \\
\hline $\begin{array}{l}\text { Mixed GCT w/ germinoma } \\
\text { component }\end{array}$ & 10 (12.5) & $24(15.6)$ & 0.53 \\
\hline $\begin{array}{l}\text { Mixed GCT w/o germinoma } \\
\text { component }\end{array}$ & $1(1.3)$ & $2(1.3)$ & 0.97 \\
\hline GCT NOS & $11(13.8)$ & $1(0.6)$ & $<0.0001^{*}$ \\
\hline \multicolumn{4}{|l|}{ Location, \% } \\
\hline Neurohypophyseal & $17(21.3)$ & $20(13.0)$ & 0.10 \\
\hline Pineal & $40(50.0)$ & $81(52.6)$ & 0.71 \\
\hline Bifocal & $15(18.8)$ & $9(5.8)$ & $0.002^{*}$ \\
\hline Basal ganglia & $0(0)$ & $13(8.4)$ & $0.008^{*}$ \\
\hline Multiple & $3(3.8)$ & $22(14.3)$ & $0.01^{*}$ \\
\hline Other & $5(6.3)$ & $9(5.8)$ & 0.90 \\
\hline
\end{tabular}

${ }^{*} p<0.05$

2283 included cases (Supplementary Table 1). ${ }^{1-3,5-11,14,16-18 \text {, }}$ 20-23,25-29,31-34,36,37,39-41,44,45,47-50,52-55,57-59,63-68 About half of the studies $(n=24)$ were from East Asia, as compared to 10 from Europe, 10 from North America, 3 from other Asian countries, and 4 from other regions. Among cohorts from Europe and North America, the prevalence of basal ganglia lesions was $0.0 \%-8.3 \%$ and $0.0 \%-7.7 \%$, respectively, with more than half (15 of 20) reporting no basal ganglia cases. Similarly, among articles from Asian countries other than East Asia, or from other geographic regions, the rate of basal ganglia GCT ranged from $1.1 \%$ to $8.1 \%$ and from $0.0 \%$ to $4.5 \%$, respectively. By contrast, the majority of reports published from East Asia documented basal ganglia lesions, and although the variance was wide at $0.0 \%-22.4 \%, 8$ of 24 reports documented basal ganglia prevalence in excess of $10 \%$, a significant difference, as compared to non-East Asian countries ( $p=0.001$; Fig. 4B). Total basal ganglia lesions counted by geographic region are detailed in Fig. $4 \mathrm{C}$, ranging from a maximum of $10.5 \%$ in East Asia (105/1229) to a minimum of $5 / 322$ in Europe and 8/377 in North America, a difference that was significant overall and in Bonferroni-corrected pairwise comparisons between East Asia and each of the other groups ( $\mathrm{p}<0.0001)$. In parallel, the overall prevalence of bifocal tumors was significantly higher in Europe (13.4\%), as compared to each other region $(\mathrm{p}<0.0001)$. The overall difference in location of the tumors among geographic regions was statistically significant $(\mathrm{p}<0.0001)$.

Furthermore, the tumor location distribution varied significantly among countries within the Far East region. When cases from Japan $(n=685)$ were compared to those from all other countries in the Far East (including Korea, China, and Taiwan, $\mathrm{n}=544$ ), the overall difference in tumor location distribution was significant ( $p<0.0001)$, as was the fraction of basal ganglia cases, which were significantly fewer in Japan (14.2 vs 6.1\%, p < 0.0001).

\section{Prognosis}

Survival endpoints were compared between the 2 databases, selecting for treatments including both platinumbased chemotherapy and radiation therapy with ventricular coverage. Germinomas meeting criteria included 23 patients from the Mayo Clinic database and 64 from the NCC, while NGGCT (excluding mature teratoma) accounted for an additional 12 Mayo Clinic cases and 38 from the NCC. The median total follow-up duration at the Mayo Clinic was 2492 days (range 43-9274 days) versus 2090 days (range 3-9488 days) in the NCC database. Among germinomas, differences in both progression-free survival (PFS) and overall survival (OS) were nonsignificant. Mayo Clinic and NCC databases, respectively, demonstrated 10-year PFS rates of $100.0 \%$ and $86.9 \%(\mathrm{p}=$ $0.08)$, and 10-year OS rates of $100 \%$ and $97.8 \%(\mathrm{p}=0.52$; Fig. 5A and B).

By contrast, a significant difference was noted between the databases for PFS in NGGCT, but not OS. More specifically, the 1-, 3-, 5-, and 10-year PFS rates in the Mayo Clinic series were $81.8 \%, 40.9 \%, 40.9 \%$, and $40.9 \%$, versus $84.3 \%, 84.3 \%, 84.3 \%$, and $75.9 \%$ in the NCC series, a difference that was statistically significant $(\mathrm{p}=0.04)$. The 1-, 3-, 5-, and 10-year OS rates in the Mayo Clinic series were $100.0 \%, 81.8 \%, 81.8 \%$, and $81.8 \%$, which were not significantly different than the NCC results at the same time points $(84.3 \%, 84.3 \%, 80.5 \%$, and $80.5 \%, \mathrm{p}=0.92$; Fig. 5C and D).

\section{Discussion}

We present a unique geographic analysis of intracranial GCT between a predominantly Caucasian cohort of patients treated at the Mayo Clinic and an almost completely Japanese cohort derived from the iGCT Consortium (NCC Japan database). Cumulatively, 234 cases were retrospectively reviewed, in an analysis that revealed no statistically significant differences in age, sex, tumor markers, histological variations, $\mathrm{PFS} / \mathrm{OS}$ in germinomas, or OS in NGGCTs, with notable significant findings in both tumor location and PFS among NGGCTs. These findings were further supported by our systematic literature review, which similarly identified a significant difference in tumor location by geographic region-in particular, with respect to basal ganglia or bifocal GCTs. 
A

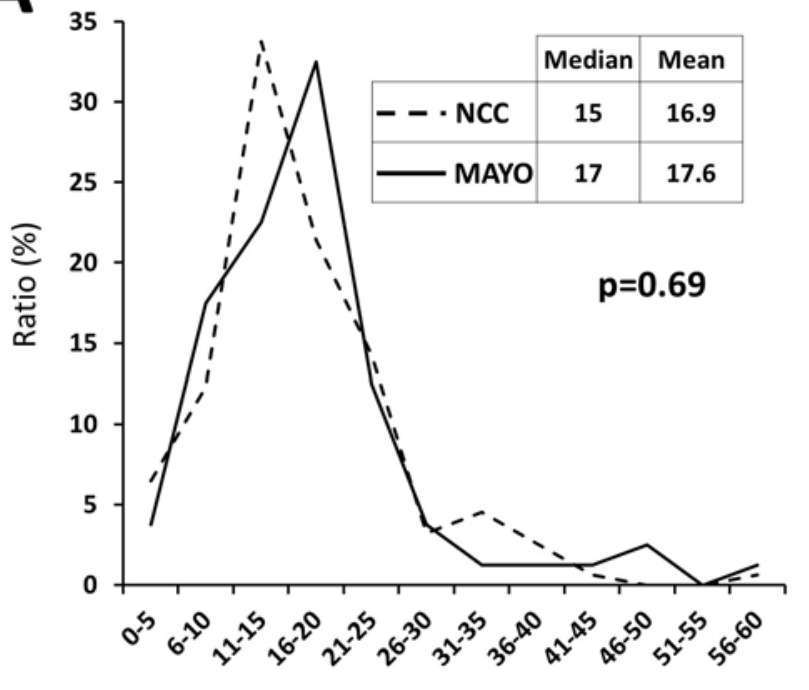

Age (years)
B
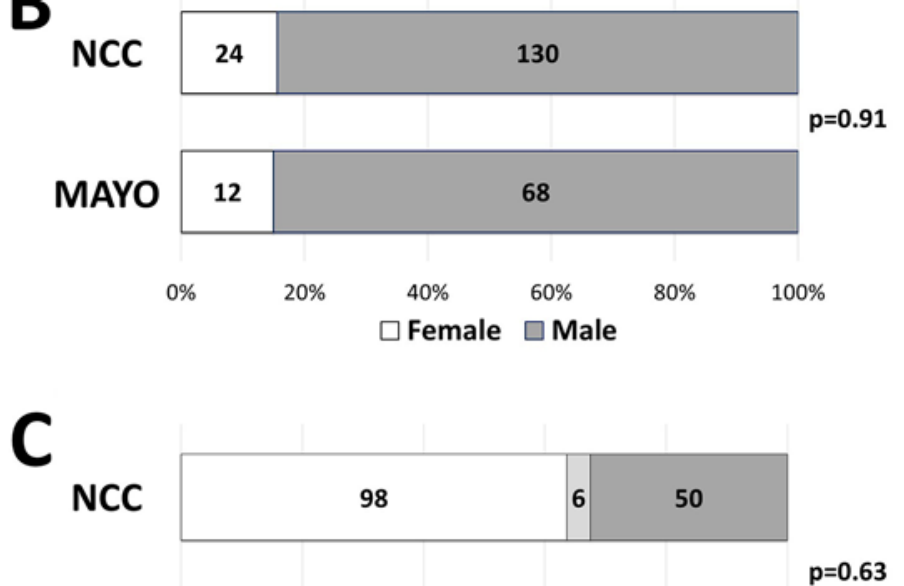

MAYO

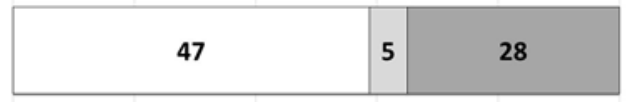

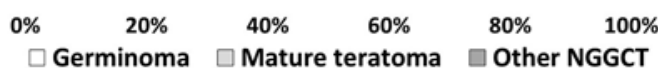

FIG. 2. A: The distribution of cases depending on age in 2 cohorts is shown. Although there is a trend of older patient age in the Mayo Clinic cohort, the distribution was not statistically significant ( $p=0.69$, Student t-test). B: The ratio of females to males was not statistically significant between the 2 cohorts ( $p=0.91$, Pearson's chi-square test). C: The ratio of germinoma, mature teratoma, and NGGCTs between the 2 cohorts showed no statistically significant difference ( $p=0.63$, Pearson's chi-square test).

Systemic GCTs arise in the midline, with primary origination at the gonads (testis, ovary) or mediastinum..$^{42}$ The GCT cell of origin is believed to be the PGC, which migrates from the base of the allantoic membrane of the yolk sac along the dorsal mesentery to the gonadal ridge during weeks 4-8 of gestation. Malignant transformation within these germ cell populations is thought to occur during the early differentiation phases of embryologic development. Of particular interest, mismigration of transformed PGCs into extragonadal organs, including the CNS, is considered to be the source of intracranial GCTs. ${ }^{61}$ Although it is considered that this mismigration of PGCs is the cause of the tumorigenesis, the reason for the predilection of the tumor occurrence for other midline sites within the CNS, including the neurohypophysis and pineal gland, remains poorly understood. Our novel observation that basal ganglia GCT incidence is variably distributed among geographic and national groups may reflect a key difference in tumor biology; we recently found that basal ganglia GCTs demonstrate a higher prevalence of mutations in the $\mathrm{PI} 3 \mathrm{~K} / \mathrm{mTOR}$ pathway than GCTs in other locations. ${ }^{60}$

Regarding lesion multiplicity, we noted that the difference was markedly decreased when bifocal cases were considered together with multifocal cases. This is most likely explained by variation in case distribution over time. More specifically, $42.5 \%$ of the pertinent Mayo Clinic cases occurred in 2000 or earlier, as compared to $23.4 \%$ of NCC database patients; correspondingly, differences in imaging technology may be responsible for a larger fraction of Mayo Clinic cases having been identified as bifocal, rather than multifocal.

We anticipate that the difference in tumor location will be better informed by future genomic investigations that build on the comparison between these groups. Given the significantly increased prevalence of GCT in general and basal ganglia GCT within the East Asian population, we also emphasize the importance of continuing a focused evaluation into the mechanisms driving neoplastic transformation in this critical patient cohort.

Of further clinical interest is the trend we observed toward improved treatment response in germinoma cases treated at the Mayo Clinic, as compared to those captured by the NCC database, which included 8 cases of recurrence, 5 of which demonstrated multiple lesions. All of these cases received standard-of-care treatment with either PE (cisplatin + etoposide, $\mathrm{n}=6$ ), CARE (carboplatin + etoposide, $\mathrm{n}=1$ ), or ICE (ifosfamide + carboplatin + etoposide, $\mathrm{n}=1$ ). However, a notable difference in treatment was identified in the radiation strategy, with almost all Mayo Clinic cases receiving whole-brain (WB) radiotherapy, while 2 underwent whole-ventricle (WV) radiotherapy, following the COG protocol (currently ACNS1123); in contrast, the majority of NCC patients underwent WV radiotherapy only (49/64). Although this finding is of academic interest, we excluded it from the principal results for several reasons, including the nonsignificant differences in response rate, the failure of prior controlled trials to demonstrate a significant improvement in association with WB over WV radiotherapy, and the absence of location-specific detail regarding recurrences in the databases we studied. Correspondingly, our conclusion is that these nonsignificant differences simply reflect normal statistical variation between ostensibly heterogeneous cohorts.

The observation of a significant difference in PFS but not OS in NGGCT warrants some further consideration. In the NCC cohort, patients were overwhelmingly treated 


\section{Serum HCG}

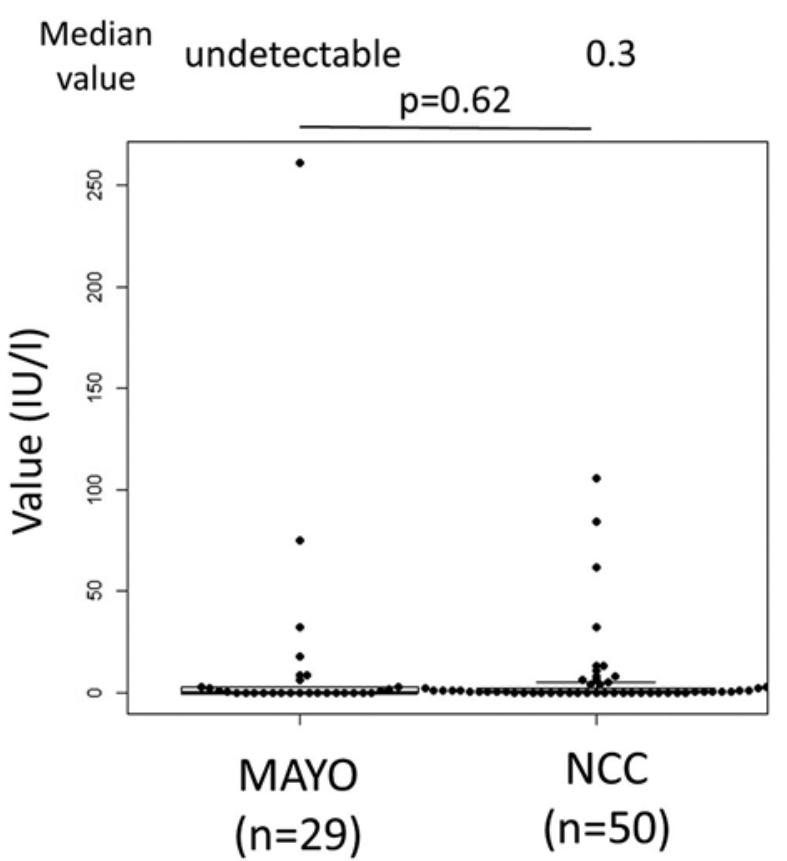

CSF HCG

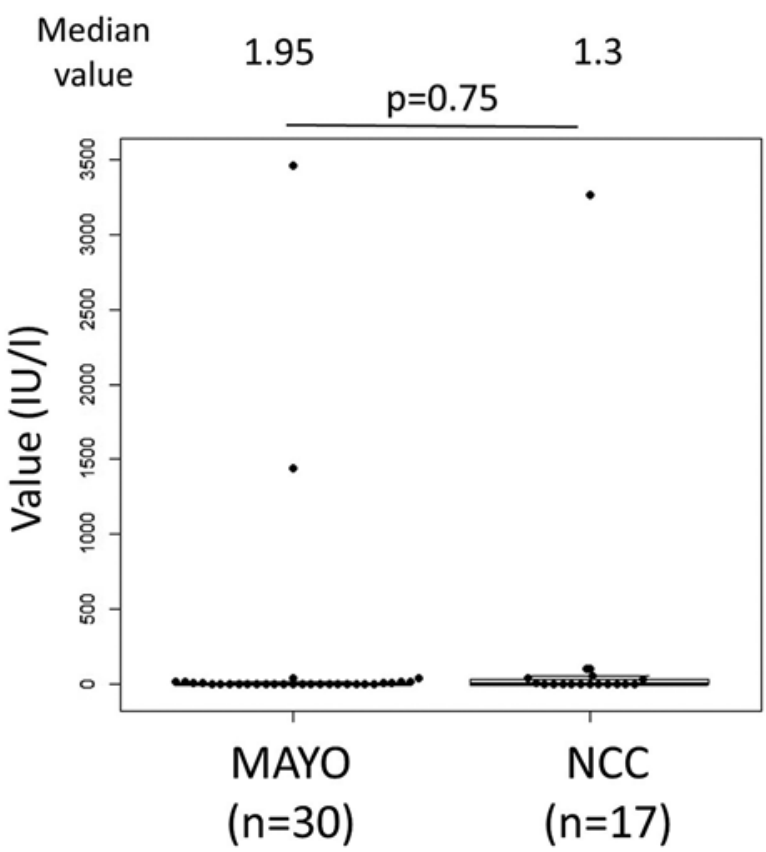

\section{Serum AFP}

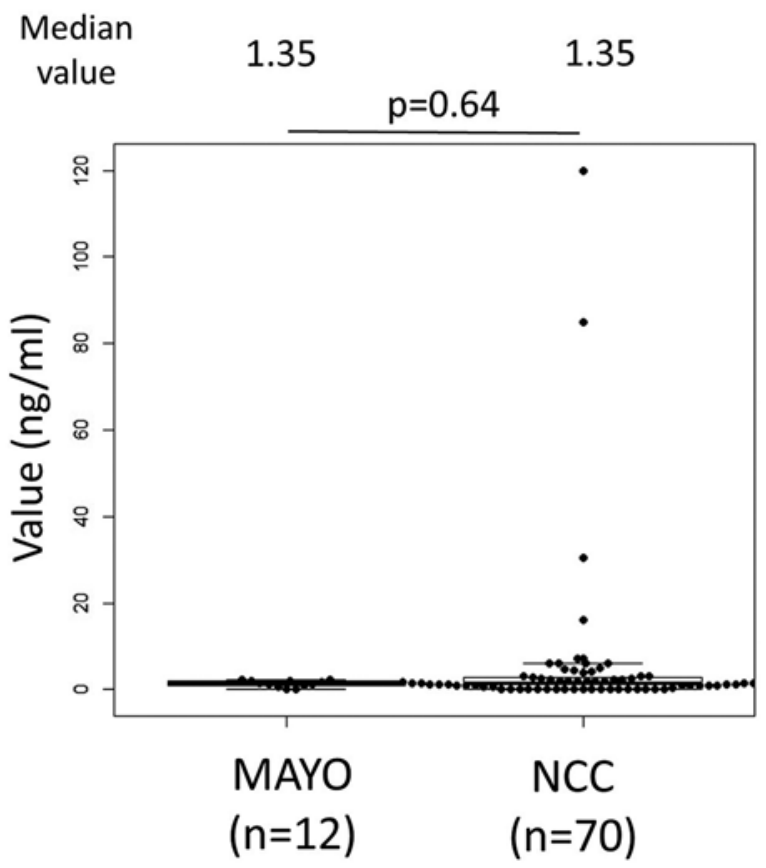

\section{CSF AFP}

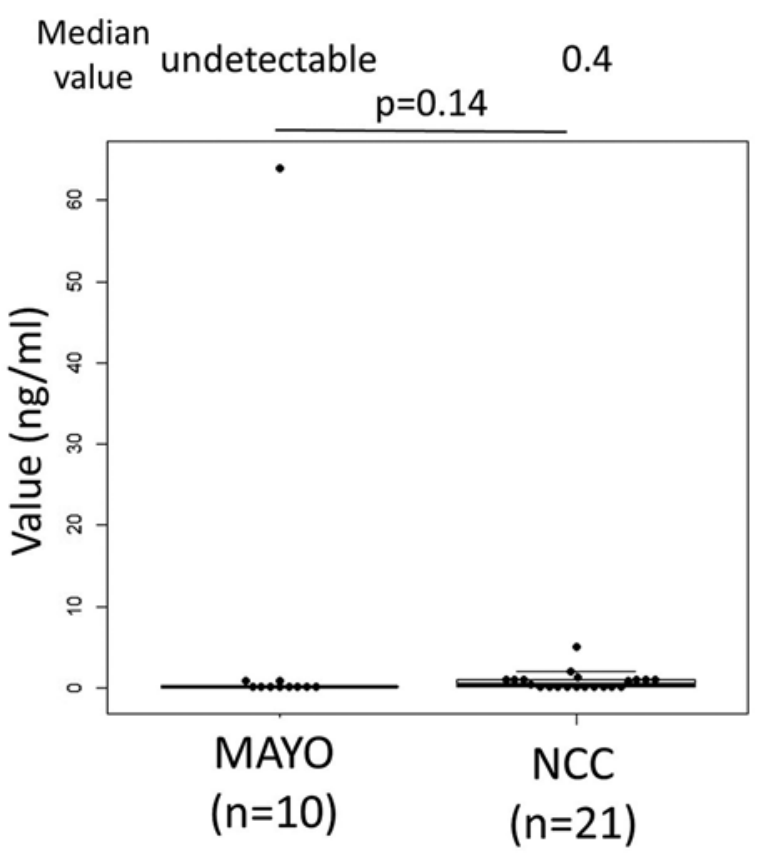

FIG. 3. Serum and CSF HCG and AFP were compared in germinoma cases between the Mayo Clinic database $(n=36)$ and NCC database $(n=79)$. There was no statistically significant difference between the 2 cohorts.

with either CARE regimen chemotherapy followed by WV radiation with a local boost for tumors in the intermediate prognosis group (e.g., immature teratoma, or mixed GCTs predominantly consisting of teratoma or germinoma components), or the ICE regimen followed by craniospinal irradiation with a local boost in the poor prognosis group (other NGGCTs except for mature teratoma). ${ }^{34}$ In the companion Mayo Clinic cohort, patients were treated using either an institutional protocol of 4 cycles of cisplatin and etoposide, or COG ACNS 0122, a protocol alternating VP-16/carboplatin with VP-16/ifosfamide for 6 cycles, followed by craniospinal irradiation with a local 
A

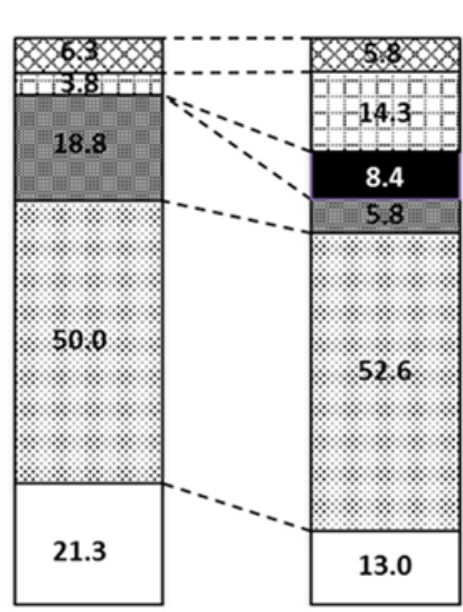

MAYO

NCC $\square$ Pineal

- Basal ganglia

$\otimes$ Other location

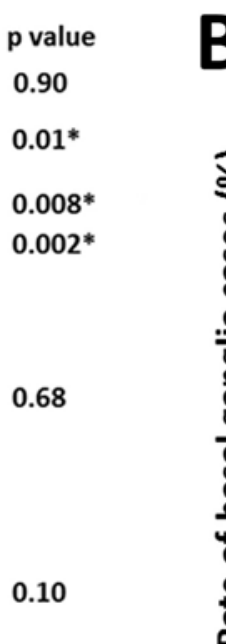

B

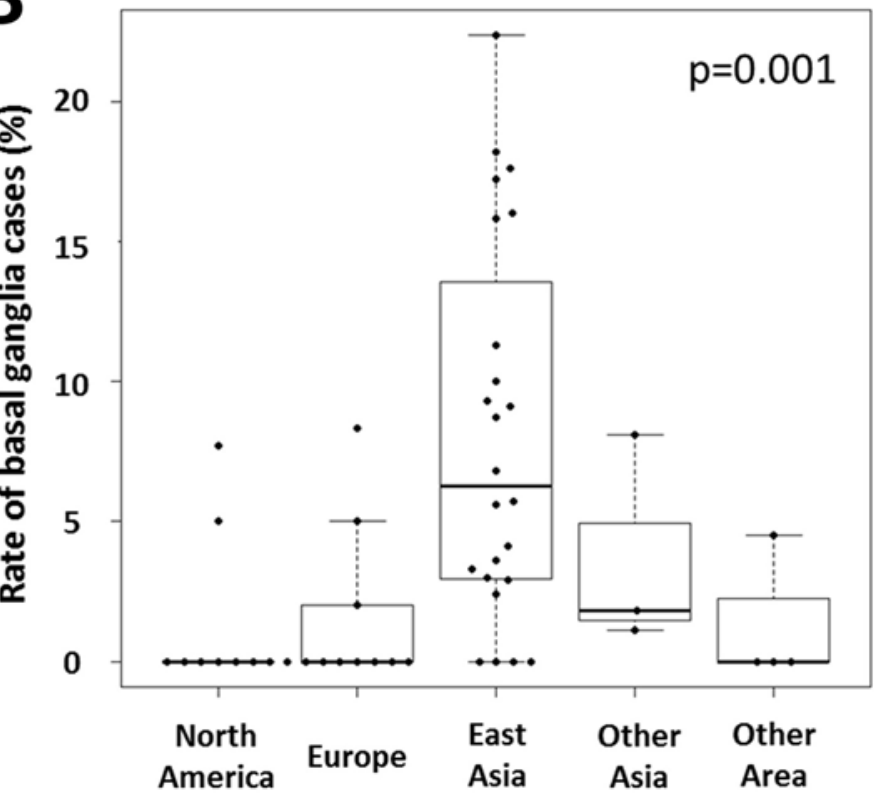

C

Europe

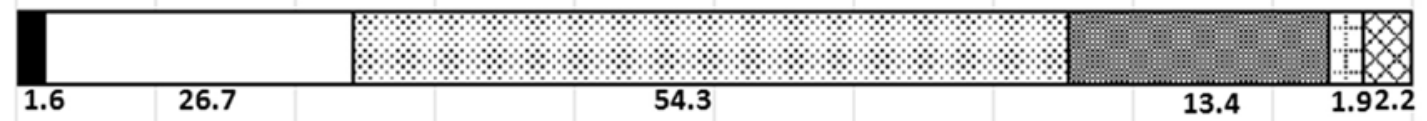

North America

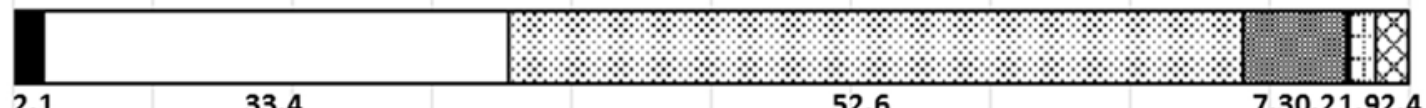

East Asia

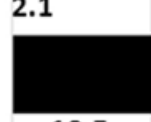

33.4

52.6

7.30.21.92.4

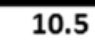

28.2

42.4

$\begin{array}{llll}8.2 & 1.04 .3 & 5.4\end{array}$

Other Asia
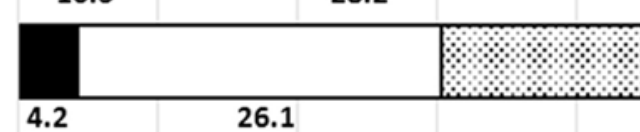

26.1

54.8

$\begin{array}{lll}3.4 & 2.3 \quad 9.2\end{array}$

Other Area
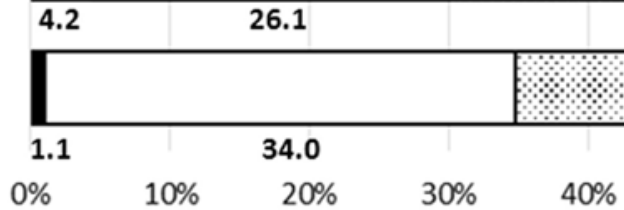

$30 \%$

$\mathbf{5 0 . 0}$

10.61.12.12.1

$0 \% \quad 10 \% \quad 20 \%$

$40 \% \quad 50 \%$

$60 \%$

$70 \%$

$90 \%$

$100 \%$

\section{Basal ganglia \\ $\square$ Neurohypophysis $\mathrm{Q}$ Pineal gland \\ Bifocal \\ 目Thalamus \\ Multiple \\ QOthers}

FIG. 4. A: The distribution of locations of tumors in each cohort is displayed. Whereas the Mayo Clinic cohort had a significantly higher rate of bifocal lesions ( $p=0.017$, Pearson's chi-square test), the NCC cohort had a significantly higher rate of basal ganglia lesions $(p=0.03)$ and multiple lesions $(p=0.016)$. B: In a systematic review of 51 reports on intracranial GCTs around the world, reports from countries in East Asia showed a significantly higher rate of GCTs in the basal ganglia compared with reports from other regions ( $p=0.001$, Wilcoxon rank-sum test). C: The distribution of tumor locations was displayed according to the geographic regions. Whereas the basal ganglia lesion rate in East Asia is $10.5 \%$, those in other regions are statistically low, i.e., $1.6 \%$ in Europe, $2.1 \%$ in North America, $4.2 \%$ in other Asia, and $1.1 \%$ in other areas.

boost. The results we observed in both cohorts are largely in accordance with those of the major preceding large studies, including the data put forth by the BTRJ, which demonstrated a 76.1\% 5-year PFS in mixed GCTs. ${ }^{56}$ When compared to the $84.3 \%$ we observed in the present study, this difference is quite modest, and likely accounted for by their exclusion of single-histology cases.$^{56}$ Comparable results were also observed in the recent SIOP (Société Inter- 


\section{A}

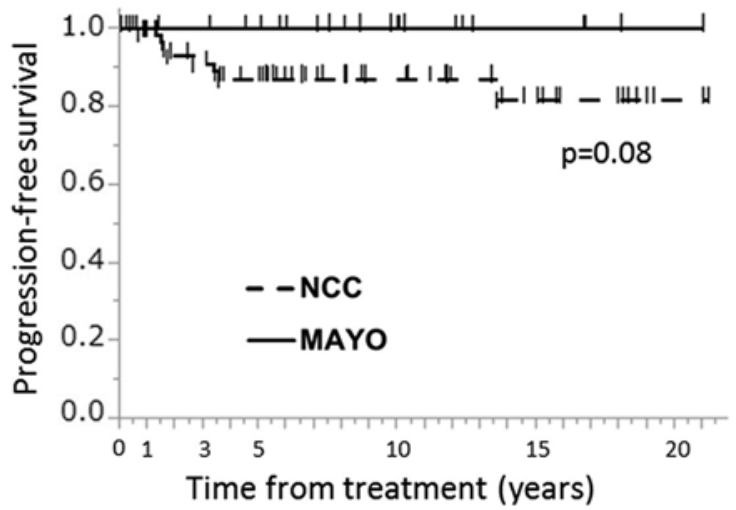

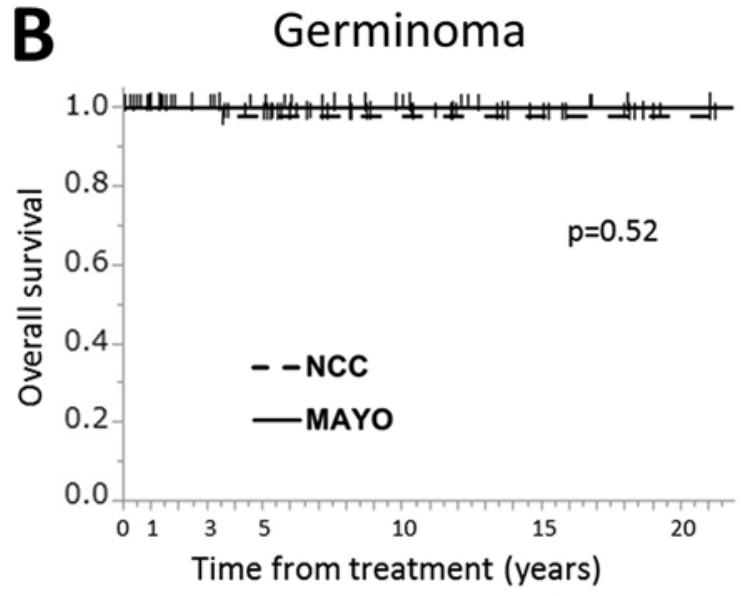

$\begin{array}{lccccccc}\text { NCC } & 63 & 57 & 48 & 42 & 24 & 13 & 3 \\ \text { MAYO } & 23 & 22 & 21 & 19 & 11 & 5 & 2 \\ & & & & & \text { Number at risk } & & \end{array}$

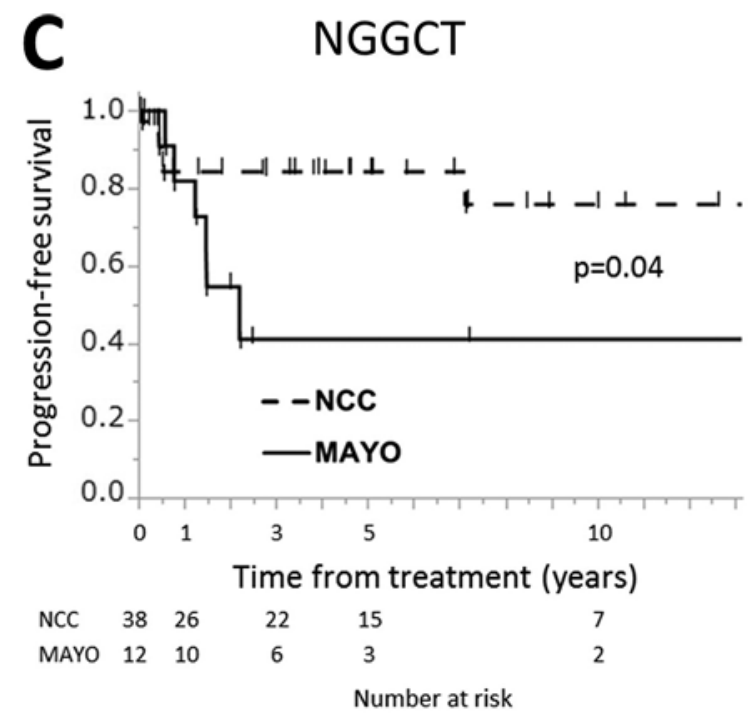

$\begin{array}{lllllccc}\text { NCC } & 63 & 57 & 48 & 42 & 24 & 13 & 3 \\ \text { MAYO } & 23 & 22 & 21 & 19 & 11 & 5 & 2\end{array}$

\section{NGGCT}

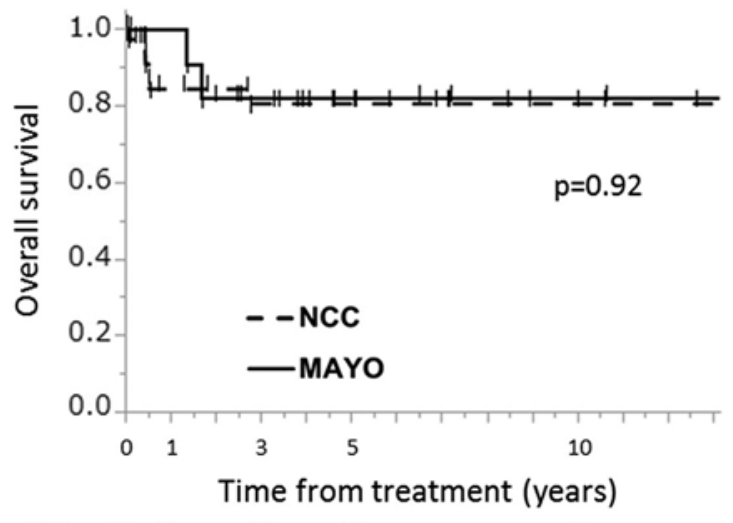

FIG. 5. A: PFS of germinoma in 2 cohorts is shown. Despite a tendency of shorter PFS observed in the NCC cohort, there was no statistically significant difference $(p=0.08$, log-rank test). Five-year PFS was $100 \%$ and $90.1 \%$ in the Mayo Clinic and NCC databases, respectively. B: OS of germinoma in the 2 cohorts shows similar results: 5 -year OS was $100 \%$ and $97.2 \%$ in the Mayo Clinic and NCC databases, respectively. C: PFS in NGGCTs in the 2 cohorts is shown. Cases in the Mayo Clinic cohort showed significantly worse prognosis than those in the NCC cohort ( $p=0.04$ ): 5 -year OS was $40.9 \%$ and $84.0 \%$ in the Mayo Clinic and NCC databases, respectively. D: OS in NGGCT in the 2 cohorts is shown. The difference between the cohorts was not statistically significant $(p=0.92): 5$-year OS was $81.8 \%$ and $79.0 \%$ in the Mayo Clinic and NCC databases, respectively.

nationale d'Oncologie Pédiatrique) study in Europe, where the 5-year PFS rate of NGGCTs was $68 \%-72 \%,{ }^{4}$ and in the COG in North America, which reported 84\% 5-year PFS. ${ }^{15}$ The result was also examined from the perspective of extent of resection in the initial stage of the treatment (Mayo Clinic vs NCC; the biopsy rate was 18.2 vs $13.2 \%$, $\mathrm{p}=0.65$, and the gross-total resection rate was $71.1 \%$ vs $63.6 \%, p=0.72$ ). Considering our results within these contexts, we note that the observed differences in PFS among NGGCTs between the 2 cohorts are unlikely to reflect inherent differences tied to the patients' racial backgrounds (and therefore genetic predispositions), but more likely to be a reflection of increased statistical variability in the small Mayo Clinic NGGCT cohort (12 cases). Previously the prognosis of GCTs was shown to be similar between two databases in the US and Japan, although the study was limited by the insufficient details of the treatment regimens. ${ }^{35}$

The present study is subject to several important limitations. As with any retrospective institutional analyses, selection, treatment, and referral biases may represent uncontrolled sources of confounding. Furthermore, although the reliance on large, prospective databases such as those maintained by the Mayo Clinic or the NCC enhances reliability within the dataset, they also restrict analysis to those endpoints included within the design of the registries. With specific respect to the NCC, case registration was predominantly motivated by genomic analyses, ${ }^{12,13,19}$ 
and correspondingly enrollment is likely biased toward cases amenable to surgical intervention and therefore specimen acquisition was performed, to the exclusion of those cases that were radiographically or biochemically diagnosed and treated empirically. With regard to the neuroanatomical distribution of basal ganglia cases, there is a potential bias toward overestimation associated with our methodological decision to limit the inclusion criteria of the systematic review to those articles precisely specifying tumor locations. Finally, although the registries both captured extensive case details, and analyzed the prognosis of the cases that were treated similarly with platinum-based chemotherapy and radiation therapy, the multicenter study design resulted in at least moderate heterogeneity with respect to treatment protocols, a potential source of confounding that we have attempted to analyze and integrate into our assessment, but that cannot be fully delineated using the available data.

\section{Conclusions}

We report the first study to compare the characteristics of intracranial GCTs between large, prospectively followed cohorts diagnosed and treated in North America and Japan. Our results build on the preceding findings of distinct genetic signatures across these populations, by highlighting novel differences in overall disease prevalence, and distribution by location within the neuraxis. We anticipate that these findings will inform future investigations into the differential origination of GCTs in these key populations, as well as the development of population-specific paradigms for treatment optimization.

\section{References}

1. Aihara Y, Watanabe S, Amano K, Komatsu K, Chiba K, Imanaka K, et al: Placental alkaline phosphatase levels in cerebrospinal fluid can have a decisive role in the differential diagnosis of intracranial germ cell tumors. J Neurosurg 131:687-694, 2018

2. Aoyama H, Shirato H, Ikeda J, Fujieda K, Miyasaka K, Sawamura Y: Induction chemotherapy followed by low-dose involved-field radiotherapy for intracranial germ cell tumors. J Clin Oncol 20:857-865, 2002

3. Baranzelli M, Patte C, Bouffet E, Portas M, MechinaudLacroix F, Sariban E, et al: An attempt to treat pediatric intracranial $\alpha \mathrm{FP}$ and $\beta \mathrm{HCG}$ secreting germ cell tumors with chemotherapy alone. SFOP experience with 18 cases. J Neurooncol 37:229-239, 1998

4. Calaminus G, Frappaz D, Kortmann RD, Krefeld B, Saran F, Pietsch T, et al: Outcome of patients with intracranial nongerminomatous germ cell tumors-lessons from the SIOPCNS-GCT-96 trial. Neuro Oncol 19:1661-1672, 2017

5. Chen MJ, da Silva Santos A, Sakuraba RK, Lopes CP, Gonçalves VD, Weltman E, et al: Intensity-modulated and 3Dconformal radiotherapy for whole-ventricular irradiation as compared with conventional whole-brain irradiation in the management of localized central nervous system germ cell tumors. Int J Radiat Oncol Biol Phys 76:608-614, 2010

6. Chung KHC, Owler BK, Dexter M, Chaseling R: Paediatric germ cell tumours of the central nervous system: results and experience from a tertiary-referral paediatric institution in Australia. J Clin Neurosci 20:514-519, 2013

7. Cormenzana Carpio M, Nehme Álvarez D, Hernández Marqúes C, Pérez Martínez A, Lassaletta Atienza A, Madero
López L: Intracranial germ cell tumours: a 21-year review. An Pediatr (Barc) 86:20-27, 2017 (Spanish)

8. Coutinho V, Dellatolas G, Castaignede-Lalande C, LongaudVales A, Kieffer V, Guerrini-Rousseau L, et al: Cognitive profile of children with intracranial germ cell tumor according to tumor location. J Pediatr Hematol Oncol 40:e424e428, 2018

9. Duron L, Sadones F, Thiesse P, Cellier C, Alapetite C, Doz $\mathrm{F}$, et al: Loco-regional extensions of central nervous system germ cell tumors: a retrospective radiological analysis of 100 patients. Neuroradiology 60:27-34, 2018

10. Feng X, Zhang S, Ichikawa T, Koga H, Washiyama K, Motoyama T, et al: Intracranial germ cell tumors: detection of p53 gene mutations by single-strand conformation polymorphism analysis. Jpn J Cancer Res 86:555-561, 1995

11. Foo AS, Lim C, Chong DQ, Tan DY, Tham CK: Primary intracranial germ cell tumours: experience of a single SouthEast Asian institution. J Clin Neurosci 21:1761-1766, 2014

12. Fukushima S, Otsuka A, Suzuki T, Yanagisawa T, Mishima K, Mukasa A, et al: Mutually exclusive mutations of KIT and RAS are associated with KIT mRNA expression and chromosomal instability in primary intracranial pure germinomas. Acta Neuropathol 127:911-925, 2014

13. Fukushima S, Yamashita S, Kobayashi H, Takami H, Fukuoka K, Nakamura T, et al: Genome-wide methylation profiles in primary intracranial germ cell tumors indicate a primordial germ cell origin for germinomas. Acta Neuropathol 133:445-462, 2017

14. Gao Y, Jiang J, Liu Q: Clinicopathological and immunohistochemical features of primary central nervous system germ cell tumors: a 24-years experience. Int J Clin Exp Pathol 7:6965-6972, 2014

15. Goldman S, Bouffet E, Fisher PG, Allen JC, Robertson PL, Chuba PJ, et al: Phase II trial assessing the ability of neoadjuvant chemotherapy with or without second-look surgery to eliminate measurable disease for nongerminomatous germ cell tumors: a Children's Oncology Group Study. J Clin Oncol 33:2464-2471, 2015

16. Haupt C, Ancker U, Müller M, Herrmann HD, Schulte FJ: Intracranial germ-cell tumours - treatment results and residuals. Eur J Pediatr 155:230-236, 1996

17. Hoei-Hansen CE, Sehested A, Juhler M, Lau YF, Skakkebaek NE, Laursen H, et al: New evidence for the origin of intracranial germ cell tumours from primordial germ cells: expression of pluripotency and cell differentiation markers. J Pathol 209:25-33, 2006

18. Hu M, Guan H, Lau CC, Terashima K, Jin Z, Cui L, et al: An update on the clinical diagnostic value of $\beta$-hCG and $\alpha$ FP for intracranial germ cell tumors. Eur J Med Res 21:10, 2016

19. Ichimura K, Fukushima S, Totoki Y, Matsushita Y, Otsuka A, Tomiyama A, et al: Recurrent neomorphic mutations of MTOR in central nervous system and testicular germ cell tumors may be targeted for therapy. Acta Neuropathol 131:889-901, 2016

20. Iwato M, Tachibana O, Tohma Y, Arakawa Y, Nitta H, Hasegawa M, et al: Alterations of the INK4a/ARF locus in human intracranial germ cell tumors. Cancer Res 60:21132115, 2000

21. Jaing TH, Wang HS, Hung IJ, Tseng CK, Yang CP, Hung PC, et al: Intracranial germ cell tumors: a retrospective study of 44 children. Pediatr Neurol 26:369-373, 2002

22. Kakkar A, Biswas A, Kalyani N, Chatterjee U, Suri V, Sharma MC, et al: Intracranial germ cell tumors: a multiinstitutional experience from three tertiary care centers in India. Childs Nerv Syst 32:2173-2180, 2016

23. Kanamori M, Kumabe T, Saito R, Yamashita Y, Sonoda Y, Ariga H, et al: Optimal treatment strategy for intracranial germ cell tumors: a single institution analysis. J Neurosurg Pediatr 4:506-514, 2009 
24. Kang JM, Ha J, Hong EK, Ju HY, Park BK, Shin SH, et al: A nationwide, population-based epidemiologic study of childhood brain tumors in Korea, 2005-2014: a comparison with United States data. Cancer Epidemiol Biomarkers Prev 28:409-416, 2019

25. Kang MJ, Kim SM, Lee YA, Shin CH, Yang SW, Lim JS: Risk factors for osteoporosis in long-term survivors of intracranial germ cell tumors. Osteoporos Int 23:1921-1929, 2012

26. Keene D, Johnston D, Strother D, Fryer C, Carret AS, Crooks $\mathrm{B}$, et al: Epidemiological survey of central nervous system germ cell tumors in Canadian children. J Neurooncol 82:289-295, 2007

27. Khafaga Y, El Weshi A, Nazmy M, Hassounah M, Alshail E, Moussa E, et al: Intracranial germ cell tumors: a singleinstitution experience. Ann Saudi Med 32:359-365, 2012

28. Kiltie AE, Gattamaneni HR: Survival and quality of life of paediatric intracranial germ cell tumour patients treated at the Christie Hospital, 1972-1993. Med Pediatr Oncol 25:450-456, 1995

29. Kretschmar C, Kleinberg L, Greenberg M, Burger P, Holmes E, Wharam M: Pre-radiation chemotherapy with responsebased radiation therapy in children with central nervous system germ cell tumors: a report from the Children's Oncology Group. Pediatr Blood Cancer 48:285-291, 2007

30. Kuroki S, Akiyoshi M, Tokura M, Miyachi H, Nakai Y, Kimura H, et al: JMJD1C, a JmjC domain-containing protein, is required for long-term maintenance of male germ cells in mice. Biol Reprod 89:93, 2013

31. Lee D, Suh YL: Histologically confirmed intracranial germ cell tumors; an analysis of 62 patients in a single institute. Virchows Arch 457:347-357, 2010

32. MacDonald SM, Trofimov A, Safai S, Adams J, Fullerton B, Ebb D, et al: Proton radiotherapy for pediatric central nervous system germ cell tumors: early clinical outcomes. Int J Radiat Oncol Biol Phys 79:121-129, 2011

33. Makino K, Nakamura H, Yano S, Kuratsu J: Incidence of primary central nervous system germ cell tumors in childhood: a regional survey in Kumamoto prefecture in southern Japan. Pediatr Neurosurg 49:155-158, 2013

34. Matsutani M, Sano K, Takakura K, Fujimaki T, Nakamura O, Funata N, et al: Primary intracranial germ cell tumors: a clinical analysis of 153 histologically verified cases. J Neurosurg 86:446-455, 1997

35. McCarthy BJ, Shibui S, Kayama T, Miyaoka E, Narita Y, Murakami M, et al: Primary CNS germ cell tumors in Japan and the United States: an analysis of 4 tumor registries. Neuro Oncol 14:1194-1200, 2012

36. Mei K, Liu A, Allan RW, Wang P, Lane Z, Abel TW, et al: Diagnostic utility of SALL4 in primary germ cell tumors of the central nervous system: a study of 77 cases. Mod Pathol 22:1628-1636, 2009

37. Morana G, Alves CA, Tortora D, Finlay JL, Severino M, Nozza P, et al: T2*-based MR imaging (gradient echo or susceptibility-weighted imaging) in midline and off-midline intracranial germ cell tumors: a pilot study. Neuroradiology 60:89-99, 2018

38. Narita Y, Shibui S: Trends and outcomes in the treatment of gliomas based on data during 2001-2004 from the Brain Tumor Registry of Japan. Neurol Med Chir (Tokyo) 55:286295,2015

39. Nishizaki T, Kajiwara K, Adachi N, Tsuha M, Nakayama $\mathrm{H}$, Ohshita N, et al: Detection of craniospinal dissemination of intracranial germ cell tumours based on serum and cerebrospinal fluid levels of tumour markers. J Clin Neurosci 8:27-30, 2001

40. Odagiri K, Omura M, Hata M, Aida N, Niwa T, Ogino I, et al: Treatment outcomes, growth height, and neuroendocrine functions in patients with intracranial germ cell tumors treat- ed with chemoradiation therapy. Int J Radiat Oncol Biol Phys 84:632-638, 2012

41. Ogiwara H, Tsutsumi Y, Matsuoka K, Kiyotani C, Terashima $\mathrm{K}$, Morota N: Apparent diffusion coefficient of intracranial germ cell tumors. J Neurooncol 121:565-571, 2015

42. Oosterhuis JW, Stoop H, Honecker F, Looijenga LH: Why human extragonadal germ cell tumours occur in the midline of the body: old concepts, new perspectives. Int J Androl 30:256-264, 2007

43. Ostrom QT, Gittleman H, Liao P, Vecchione-Koval T, Wolinsky Y, Kruchko C, et al: CBTRUS Statistical Report: Primary brain and other central nervous system tumors diagnosed in the United States in 2010-2014. Neuro Oncol 19 (Suppl 5):v1-v88, 2017

44. Park Y, Yu ES, Ha B, Park HJ, Kim JH, Kim JY: Neurocognitive and psychological functioning of children with an intracranial germ cell tumor. Cancer Res Treat 49:960-969, 2017

45. Phi JH, Kim SK, Lee YA, Shin CH, Cheon JE, Kim IO, et al: Latency of intracranial germ cell tumors and diagnosis delay. Childs Nerv Syst 29:1871-1881, 2013

46. Poynter JN, Fonstad R, Tolar J, Spector LG, Ross JA: Incidence of intracranial germ cell tumors by race in the United States, 1992-2010. J Neurooncol 120:381-388, 2014

47. Qaddoumi I, Sane M, Li S, Kocak M, Pai-Panandiker A, Harreld J, et al: Diagnostic utility and correlation of tumor markers in the serum and cerebrospinal fluid of children with intracranial germ cell tumors. Childs Nerv Syst 28:10171024,2012

48. Queipo G, Aguirre D, Nieto K, Peña YR, Palma I, Olvera J, et al: Intracranial germ cell tumors: association with Klinefelter syndrome and sex chromosome aneuploidies. Cytogenet Genome Res 121:211-214, 2008

49. Raiyawa T, Khorprasert C, Lertbutsayanukul C, Seksarn P, Sosothikul D, Amornfa J, et al: Management of intracranial germ cell tumors at the King Chulalongkorn Memorial Hospital. J Med Assoc Thai 95:1327-1334, 2012

50. Rogers PB, Sims EC, Plowman N: Blood to cerebrospinal fluid human chorionic gonadotropin-beta ratios in intracranial germ cell tumors. Neurosurg Focus 5(1):e4, 1998

51. Rosen A, Jayram G, Drazer M, Eggener SE: Global trends in testicular cancer incidence and mortality. Eur Urol 60:374379, 2011

52. Saitoh M, Tamaki N, Kokunai T, Matsumoto S: Clinicobiological behavior of germ-cell tumors. Childs Nerv Syst 7:246-250, 1991

53. Sakurada K, Saino M, Mouri W, Sato A, Kitanaka C, Kayama T: Nestin expression in central nervous system germ cell tumors. Neurosurg Rev 31:173-177, 2008

54. Salzman KL, Rojiani AM, Buatti J, Quisling RG, Marcus RB Jr, Maria BL, et al: Primary intracranial germ cell tumors: clinicopathologic review of 32 cases. Pediatr Pathol Lab Med 17:713-727, 1997

55. Schneider DT, Zahn S, Sievers S, Alemazkour K, Reifenberger G, Wiestler OD, et al: Molecular genetic analysis of central nervous system germ cell tumors with comparative genomic hybridization. Mod Pathol 19:864-873, 2006

56. Shibui S: Brain Tumor Registry of Japan (2001-2004). Neurol Med Chir (Tokyo) 54 Suppl:1-102, 2013

57. Smith AA, Weng E, Handler M, Foreman NK: Intracranial germ cell tumors: a single institution experience and review of the literature. J Neurooncol 68:153-159, 2004

58. Sugiyama K, Arita K, Tominaga A, Hanaya R, Taniguchi E, Okamura T, et al: Morphologic features of human chorionic gonadotropin- or alpha-fetoprotein-producing germ cell tumors of the central nervous system: histological heterogeneity and surgical meaning. Brain Tumor Pathol 18:115-122, 2001

59. Takahashi S, Yoshida K, Kawase T: Intracranial germ cell 
tumors: efficacy of neoadjuvant chemo-radiotherapy without surgical biopsy. Keio J Med 60:56-64, 2011

60. Takami H, Fukuoka K, Fukushima S, Nakamura T, Mukasa A, Saito N, et al: Integrated clinical, histopathological, and molecular data analysis of 190 central nervous system germ cell tumors from the iGCT consortium. Neuro Oncol 21:1565-1577, 2019

61. Teilum G: Classification of endodermal sinus tumour (mesoblatoma vitellinum) and so-called "embryonal carcinoma" of the ovary. Acta Pathol Microbiol Scand 64:407-429, 1965

62. Wang L, Yamaguchi S, Burstein MD, Terashima K, Chang K, $\mathrm{Ng} \mathrm{HK}$, et al: Novel somatic and germline mutations in intracranial germ cell tumours. Nature 511:241-245, 2014

63. Weiner HL, Lichtenbaum RA, Wisoff JH, Snow RB, Souweidane MM, Bruce JN, et al: Delayed surgical resection of central nervous system germ cell tumors. Neurosurgery 50:727-734, 2002

64. Wolden SL, Wara WM, Larson DA, Prados MD, Edwards MS, Sneed PK: Radiation therapy for primary intracranial germ-cell tumors. Int J Radiat Oncol Biol Phys 32:943949, 1995

65. Wu CC, Guo WY, Chang FC, Luo CB, Lee HJ, Chen YW, et al: MRI features of pediatric intracranial germ cell tumor subtypes. J Neurooncol 134:221-230, 2017

66. Yang JC, Terezakis SA, Dunkel IJ, Gilheeney SW, Wolden SL: Intensity-modulated radiation therapy with dose painting: a brain-sparing technique for intracranial germ cell tumors. Pediatr Blood Cancer 63:646-651, 2016

67. Yoshida J, Sugita K, Kobayashi T, Takakura K, Shitara N, Matsutani M, et al: Prognosis of intracranial germ cell tumours: effectiveness of chemotherapy with cisplatin and etoposide (CDDP and VP-16). Acta Neurochir (Wien) 120:111-117, 1993

68. Zissiadis Y, Dutton S, Kieran M, Goumnerova L, Scott RM, Kooy HM, et al: Stereotactic radiotherapy for pediatric intracranial germ cell tumors. Int J Radiat Oncol Biol Phys 51:108-112, 2001

\section{Disclosures}

The authors report no conflict of interest concerning the materials or methods used in this study or the findings specified in this paper.

\section{Author Contributions}

Conception and design: Daniels, Takami, Perry, Graffeo, Nishikawa. Acquisition of data: Takami, Giannini. Analysis and interpretation of data: Takami. Drafting the article: Takami. Critically revising the article: Daniels, Takami, Perry, Graffeo, Narita, Nakazato, Saito, Nishikawa, Matsutani, Ichimura.

Reviewed submitted version of manuscript: all authors. Approved the final version of the manuscript on behalf of all authors: Daniels. Statistical analysis: Takami. Administrative/technical/ material support: Daniels, Perry, Graffeo. Study supervision: Daniels, Nishikawa, Matsutani, Ichimura.

\section{Supplemental Information Online-Only Content}

Supplemental material is available with the online version of the article.

Supplementary Table 1. https://thejns.org/doi/suppl/10.3171/ 2019.11.JNS191576.

\section{Correspondence}

David J. Daniels: Mayo Clinic, Rochester, MN. daniels.david@ mayo.edu. 